\title{
Esophageal motility abnormalities in Egyptian patients using high resolution esophageal manometry: a descriptive study
}

\author{
Gina Gamal Naguib* (D), Mohamed Hassan, Ahmed I. Elshafie and M. G. Naguib
}

\begin{abstract}
Background and aim: For many years, esophageal manometry has been used for assessment of upper gastro-intestinal (GI) symptoms. Chicago classification is the key for diagnosis and managing motility disorders as it is considered as a standardized approach for categorization of esophageal abnormalities. The aim of this study is to analyze types of esophageal motility findings in Egyptian cases who were suffering from upper Gl complaints. Methods: This descriptive study included 378 subjects who were suffering from upper $\mathrm{Gl}$ complaints as dysphagia, vomiting, chest pain and regurgitation in the period between 10/2015-7/2020. Esophageal HRM study was performed for all patients (MMS Laborie device). The catheter was positioned and confirmed passing across the EGJ (esophago-gastric junction) using landmarks. Swallows and resting status were recorded. Anatomical landmarks were placed.

Results: Most of the patients were complaining of upper Gl symptoms. Males were $49.2 \%$ of cases. Mean age was 41.3. Dysphagia was the prominent symptom while chest pain was the least symptom. Many manometry findings were observed including ineffective motility, achalasia, absent contractility, EGJ outflow obstruction, jackhammer esophagus and normal findings. Type II achalasia was the dominant type in achalasia patients while Type III was the least. LES was normotensive in most of the cases. Hiatus hernia $(\mathrm{HH})$ was detected in $40.2 \%$ of the cases.
\end{abstract}

Conclusion: This is considered the first Egyptian descriptive study to determine the prevalence of esophageal motility abnormalities in Egyptian patients complaining of upper Gl symptoms. HRM is very important for patients complaining of upper Gl symptoms.

Keywords: Manometry, Motility, Esophageal topography, Achalasia

\section{Background}

Esophageal manometry is an evaluation of the pressure pattern which resulted from contractions of the esophageal muscles [1]. Chest pain and dysphagia are common esophageal dysmotility manifestations [2]. Esophageal manometry is the cornerstone for assessment of esophageal motor functions as it helps in evaluation of esophageal peristalsis by measuring the pattern, force, and time taken by each swallow [3].

\footnotetext{
*Correspondence: Gina_gamal@med.asu.edu.eg

Department of Internal Medicine, Gastroenterology and Hepatology

Unit, Faculty of Medicine, Ain Shams University, Al-Abbasya square, Cairo, Egypt
}

For clinical purposes, it can be used to define the characteristics of the esophageal contractions inorder to identify pathological conditions [4]. For many years, esophageal manometry has been used for assessment of cases with upper GI complaints as dysphagia [5].

The combination of pressure topography plotting and high-resolution manometry resulted in the appearance of esophageal pressure topography (EPT) which is widely used for esophageal motility clinical evaluation due to its high resolution form [6].

HRM has more advantages than conventional manometry such as close spacing of the sensors (reaching $1 \mathrm{~cm}$ intervals), higher spatial resolution, more details of esophageal motility and easy interpretation of 
the findings owing to the colored spatiotemporal plot instead of traditional lines [7].

Objective analysis can be provided by Chicago classification for HRM metrics and topography [8]. It is the cornerstone for diagnosis and hence managing motility disorders as it is considered as a standardized approach for esophageal abnormalities [9].

By using chicago classification, HRM has superior inter-rater agreement with high accuracy in reaching the diagnosis even for non-experts $[10,11]$ and is easier to learn [12].

The aim of this study is to analyze types of esophageal motility findings in Egyptian patients who were suffering from upper GI complaints.

\section{Methods}

This descriptive study included data from patients' records who cannot be re-contacted. It included 378 cases who were suffering from upper GI complaints as dysphagia, vomiting, chest pain and regurgitation from many areas in Egypt in the period between $10 / 2015-7 / 2020$.

The study was approved by the Ethical Review Board of Ain Shams university (Reference number: FMASU R 176/2021). The study protocol conforms to the ethical guidelines of 1975 Declaration of Helsinki as reflected in a prior approval by the institution's human research committee.

Esophageal HRM study was performed for all patients (MMS Laborie device) after a $6-8 \mathrm{~h}$ fasting period. Patients were also informed to stop for a minimum of $72 \mathrm{~h}$ before the procedure any anticholinergic drugs, nitrates, calcium channel blockers and prokinetic drugs. A water state HRM catheter was used with 24 pressure channels.

Before insertion of the catheter, the catheter was adjusted to zero to the atmospheric pressure then it was placed trans-nasally while the patient was sitting down, and the catheter was positioned to record from the UES to the stomach.

Then patients were advised to lie flat and the position of the catheter was confirmed passing across the EGJ using landmarks and instructed the patient to take a deep breath to ensure that the catheter has reached the stomach.

First, participants were asked to stop swallowing for 30-60 s (baseline recordings of LES pressure) and then participants were asked to repeat swallowing $5 \mathrm{ml}$ water, at a minimum of $20 \mathrm{~s}$ intervals between each swallow.

Anatomical landmarks were placed as UES, transition zone, LES, PIP and gastric marks.

\section{Statistical analysis}

After completion of the procedure, automatic landmarks were revised and adjusted manually if an error was seen then analysis of the data recorded was done according to the Chicago classification v2.0 because it was the available software during that period.

LES was assessed by using IRP $4 \mathrm{~s}$ (integrated relaxation pressure) which was calculated by measuring the mean of the lowest LES pressure over $4 \mathrm{~s}$ which was measured in the $10 \mathrm{~s}$ interval after UES relaxation window. IRP $4 \mathrm{~s}$ was considered high if it was $\geq 15 \mathrm{mmHg}$. Evidence of $\mathrm{HH}$ was observed in cases of double humped LES.

Properties of the contraction in the esophagus were assessed using the DCI (distal contractile integral) which was calculated by multiplication of the swallow duration by esophageal smooth muscles length by pressure amplitude which was more than $20 \mathrm{mmHg}$ for assessing the occurrence of failed and weak contractions.

\section{Data management and analysis}

Data were collected and analyzed on computer using SPSS package version number 20. Quantitative data were tested for normality with Shapiro-Wilk test and described as mean, standard deviation (SD). Qualitative data were analyzed in the form of frequencies ( $\mathrm{n}$ ) and percentage (\%). Chi-square describe the association between qualitative variables. $P$-value $\leq 0.05$ was considered significant.

\section{Results}

This descriptive study included 378 patients who were complaining of upper GI symptoms. Males were $49.2 \%$ of cases. Age was widely ranging from 11 to 81 years old with mean age 41.3.

Upper GI symptoms were noticed in $98 \%$ of the patients. Dysphagia was the prominent symptom (41.3\%) while chest pain was the least symptom (3.7\%). Minority of patients $(1.8 \%)$ were asymptomatic but manometry was done either preoperatively before or after an intervention (Table 1).

Many manometry findings were observed including ineffective motility (28\%), achalasia (20.9\%), absent contractility (12.7\%), EGJ outflow obstruction (6.3\%), jackhammer esophagus $(0.8 \%)$ while normal findings (31.2\%) were also noticed (Fig. 1).

Our study revealed that the dominant type of achalasia in our patients was Type II while the least one was Type III (63.3\% versus $6.3 \%$ of the patients).

LES was normotensive in most of the cases. Hiatus hernia was detected in $40.2 \%$ of the cases (Table 2). 
Table 1 Descriptive analysis of personal characteristics of study cases

\begin{tabular}{llllll}
\hline & & Mean & \pm SD & Minimum & Maximum \\
\hline Age & & 41.38 & 13.66 & 11.00 & 81.00 \\
Sex & Male & 186 & $49.2 \%$ & & \\
\multirow{4}{*}{ Indication } & Female & 192 & $50.8 \%$ & & \\
& Dysphagia & 156 & $41.3 \%$ & & \\
& Heart burn & 95 & $25.1 \%$ & & \\
& Regurge & 65 & $17.2 \%$ & & \\
& Vomiting & 41 & $10.8 \%$ & & \\
& Chest pain & 14 & $3.7 \%$ & & \\
& postoperative & 5 & $1.4 \%$ & & \\
& Preoperative & 2 & $0.5 \%$ & & \\
\hline
\end{tabular}

$S D$ standard deviation

\section{Discussion}

This is the first study to discuss manometry findings in Egyptian population using high resolution topography. A total of 378 patients underwent high resolution esophageal manometry with wide range of age.
Most of the patients were complaining of upper GI symptoms. Dysphagia was the prominent symptom while chest pain was the least symptom. Seven patients were asymptomatic but manometry was done either preoperatively before or after an intervention (Table 1). Hiatus hernia $(\mathrm{HH})$ was detected in $40.2 \%$ of the cases.

Many manometry findings were observed including ineffective motility, achalasia, absent contractility, EGJ outflow obstruction, jackhammer esophagus and normal findings. The most common was ineffective motility followed by achalasia. While the least was jackhammer esophagus. Type II achalasia was the dominant type in achalasia patients while Type III was the least.

Achalasia was described as a common symptom by Rehman et al. [13] (35.6\% of 202 patients) and Cisternas et al. [14] (31.2\% of 426 patients).

In agreement with Serrano et al. who observed that $45 \%$ of the patients in the study were females and $55 \%$ were males, with a mean age of $61.5 \pm 16.2$ yrs. (range 20-87 years old). Many cases presented mainly by dysphagia ( $85 \%$ of cases) with some patients complained of reflux ( $45 \%$ of cases) $(n=31)$, chest pain (23\% of cases)

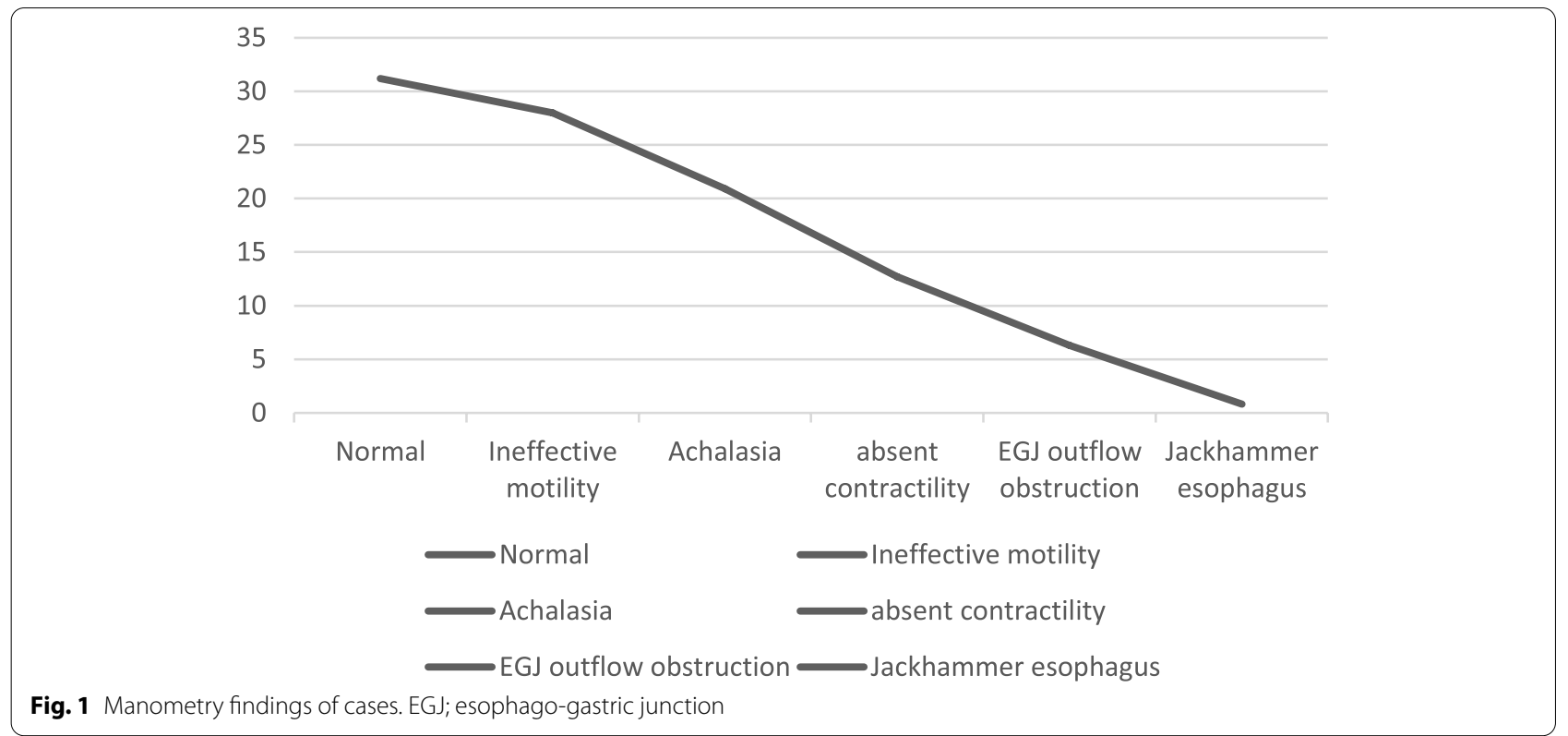

Table 2 Relation between sex and $\mathrm{HH}$

\begin{tabular}{|c|c|c|c|c|c|c|c|}
\hline & & \multicolumn{4}{|l|}{ Sex } & \multirow[t]{3}{*}{$\mathrm{P}^{*}$} & \multirow[t]{3}{*}{ Sig } \\
\hline & & \multicolumn{2}{|c|}{ Male } & \multicolumn{2}{|c|}{ Female } & & \\
\hline & & $\mathrm{n}$ & $\%$ & $\mathrm{n}$ & $\%$ & & \\
\hline \multirow[t]{2}{*}{ Hiatus Hernia } & No & 115 & $61.8 \%$ & 111 & $57.8 \%$ & 0.426 & NS \\
\hline & Yes & 71 & $38.2 \%$ & 81 & $42.2 \%$ & & \\
\hline
\end{tabular}


( $n=13)$, heartburn (13\% of cases) ( $n=9)$, weight loss $(6 \%$ of cases) $(n=4)$ and cough ( $4 \% \mathrm{f}$ cases) $(\mathrm{n}=3)$ [15].

Serrano et al. observed 51 achalasia cases (30 cases were type II, 9 were type I and 12 were type III). Six cases were jackhammer esophagus, eight cases were DES and five were esophago-gastric junction outflow obstruction [15].

In parallel to a study done at Barnes Hospital, St. Louis, Missouri, in the period between 1980 and 1982. Patients were referred for esophageal manometry. Patients, who were presented with chest pain, were evaluated (34\% of the patients), dysphagia (43\% of the patients), and heartburn ( $7 \%$ of the patients). Few patients $(9 \%)$ were referred to confirm or to exclude diagnosis of achalasia, and few (7\%) were referred for miscellaneous causes [16].

Maziak et al. studied preoperative esophageal motility in patients with paraesophageal hiatus hernia. Common symptoms were recorded as reflux symptoms $(80 \%$ of cases), postprandial pain (56\% of the cases) and heart burn (in $31 \%$ of cases). Indicators of severe reflux disease were observed as hypotensive LES (in 51\% of cases), and ineffective distal esophageal peristalsis (in 59\% of patients) [17].

Nottingham University Hospitals developed a study included patients with esophageal symptoms using a development study while a validation study was done at University Hospital Zürich (Switzerland). Many patients (48\%) were complaining of dysphagia while others (52\%) were complaining of reflux or other symptoms. The validation study included 221 patients, $44 \%$ of them had dysphagia and 56\% had symptoms of reflux [18].

Kahrilas et al. described the result of a pressure topography patterns via systematic analysis in 475 cases. Many findings were observed including aperistalsis (29 cases), achalasia (73 cases) and distal esophageal spasm (6 cases) [19].

Sixty-eight patients with GERD completed a study cohort. They included normal peristalsis (38 cases) and minor peristaltic disorders (30), mainly IEM. Many symptoms were recorded including regurgitation, chest pain, heart burn, dysphagia and other non-specific as (cough and hoarseness of voice). Regurgitation was the commonest symptom $(68.4 \%$ of cases in the normal motility group versus $87 \%$ of cases in the minor motility disorders) [20].

\section{Conclusions}

This is considered the first descriptive study to observe and describe different esophageal motility findings in Egyptian cases who were suffering from upper GI complaints. HRM is very important for cases suffering from upper GI symptoms.

\section{Abbreviations}

Gl: Gastro-intestinal; HRM: High resolution manometry; EGJ: Esophago-gastric junction; EPT: Esophageal pressure topography; LES: Lower esophageal sphincter; UES: Upper esophageal sphincter; PIP: Pressure inversion point; IRP: Integrated relaxation pressure; DCI: Distal contractile integral; SD: Standard deviation; Gerd: Gastro-esophageal reflux disease; IEM: Ineffective esophageal motility.

\section{Acknowledgements \\ None.}

Authors' contributions

GGN: conceptualization/ performing the procedure/ data collection/ revision of the manuscript. MHF: Data analysis / writing the manuscript/ revision of the manuscript. AlE: Data analysis / writing the manuscript/ revision of the manuscript. MGN: Data analysis/ writing the manuscript/ revision of the manuscript. All authors shared in finalization of the manuscript till it reached its final stage.

\section{Funding}

Not Applicable.

\section{Availability of data and materials}

The datasets used and analyzed during the current study are available from corresponding author upon reasonable request.

\section{Declarations}

\section{Ethics approval and consent to participate}

The study was approved by the Ethical Review Board of Ain Shams university (Reference number: FMASU R 176/2021). The study protocol conforms to the ethical guidelines of 1975 Declaration of Helsinki as reflected in a prior approval by the institution's human research committee. This descriptive study is a retrospective study which included data from patients' records who cannot be re-contacted.

\section{Consent for publication}

Not Applicable.

\section{Competing interests}

All authors declare that they have no competing interests.

Received: 22 September 2021 Accepted: 5 December 2021

Published online: 06 January 2022

References

1. Weijenborg PW, Kessing BF, Smout AJPM et al (2014) Normal values for solid-state esophageal high-resolution manometry in a European population; an overview of all current metrics. Neurogastroenterol Motil 26:654-659

2. Van Hoeij FB, Bredenoord AJ (2016) Clinical application of esophageal high-resolution manometry in the diagnosis of esophageal motility disorders. J Neurogastroenterol Motil 22(1):6-13

3. Savarino E, Tutuian R (2008) Combined multichannel intraluminal impedance and manometry testing. Dig Liver Dis 40:167-173

4. Gilger MA, Boyle JT, Sondheimer JM et al (1997) A Medical Position Statement of the North American Society for Pediatric Gastroenterology and Nutrition: indications for pediatric esophageal manometry. J Pediatr Gastroenterol Nutr 24:616-618

5. Spechler SJ, Castell DO (2001) Classification of oesophageal motility abnormalities. Gut 49:145-151

6. Clouse RE, Staiano A, Alrakawi A et al (2000) Application of topographical methods to clinical esophageal manometry. Am J Gastroenterol 95:2720-2730

7. Grübel C, Hiscock R, Hebbard G (2008) Value of spatiotemporal representation of manometric data. Clin Gastroenterol Hepatol 6:525-530

8. Kahrilas PJ, Bredenoord AJ, Fox M et al (2015) The Chicago classification of esophageal motility disorders, v3.0. Neurogastroenterol Motil 27(2):160-174 
9. RohofWOA, Bredenoord AJ (2017) Chicago classification of esophageal motility disorders: lessons learned. Curr Gastroenterol Rep 19:37. https:/ doi.org/10.1007/s1 1894-017-0576-7

10. Fox MR, Pandolfino JE, Sweis R et al (2015) Inter-observer agreement for diagnostic classification of esophageal motility disorders defined in highresolution manometry. Dis Esophagus 28(8):711-719

11. Carlson DA, Ravi K, Kahrilas PJ et al (2015) Diagnosis of esophageal motility disorders: esophageal pressure topography vs. conventional line tracing. Am J Gastroenterol 110(7):967-977

12. Soudagar AS, Sayuk GS, Gyawali CP (2012) Learners favour high resolution oesophageal manometry with better diagnostic accuracy over conventional line tracings. Gut 61 (6):798-803

13. Rehman H, Abid A, Awan S et al (2020) Spectrum and clinical outcome of motility disorders on high-resolution esophageal manometry: a study from a tertiary center on patients with dysphagia in Pakistan. Cureus 12(12):e12088. https://doi.org/10.7759/cureus.12088

14. Cisternas D, Taft T, Carlson DA et al (2020) Validation and psychometric evaluation of the Spanish version of brief esophageal dysphagia questionnaire (BEDQ): results of a multicentric study. Neurogastroenterol Motil 00:e14025. https://doi.org/10.1111/nmo.14025

15. Serrano L, Saad AR, DuCoin C et al (2020) Discordance between highresolution manometry, esophagoscopy and contrast esophagogram in determining landmarks for per-oral endoscopic myotomy in spastic esophageal disorders: a word of caution. Surg Endosc 13. https://doi.org/ 10.1007/s00464-020-08064-8

16. Clouse R, Staiano A (1983) Contraction abnormalities of the esophageal body in patients referred for manometry a new approach to manometric classification. Dig Dis Sci 28(9):784-791

17. Maziak DE, Todd TRJ, Pearson FG (1998) Massive hiatus hernia: evaluation and surgical management. J Thorac Cardiovasc Surg 115:53-62

18. Ang D, Misselwitz B, Hollenstein M et al (2017) Diagnostic yield of high-resolution manometry with a solid test meal for clinically relevant, symptomatic oesophageal motility disorders: serial diagnostic study. Lancet Gastroenterol Hepatol 2(9):619-690 e6

19. Kahrilas PJ, Ghosh SK, Pandolfino JE (2008) Esophageal Motility Disorders in Terms of Pressure Topography, The Chicago Classification. J Clin Gastroenterol 42(5):627-635

20. Jain M, Agrawal V (2021) Evaluation of esophageal motor function in patients with gastroesophageal reflux using multiple rapid swallows. Indian J Gastroenterol. https://doi.org/10.1007/s12664-020-01124-w

\section{Publisher's Note}

Springer Nature remains neutral with regard to jurisdictional claims in published maps and institutional affiliations.

\section{Submit your manuscript to a SpringerOpen ${ }^{\circ}$ journal and benefit from:}

- Convenient online submission

- Rigorous peer review

- Open access: articles freely available online

- High visibility within the field

- Retaining the copyright to your article

Submit your next manuscript at $\boldsymbol{\nabla}$ springeropen.com 